\title{
Witzel, Marc
}

\section{Sozialpädagogische Orte im digitalen Raum}

Wunder, Maik [Hrsg.]: Digitalisierung und Soziale Arbeit. Transformationen und Herausforderungen. Bad Heilbrunn : Verlag Julius Klinkhardt 2021, S. 68-79

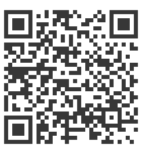

Quellenangabe/ Reference:

Witzel, Marc: Sozialpädagogische Orte im digitalen Raum - In: Wunder, Maik [Hrsg.]: Digitalisierung und Soziale Arbeit. Transformationen und Herausforderungen. Bad Heilbrunn : Verlag Julius Klinkhardt 2021, S. 68-79 - URN: urn:nbn:de:0111-pedocs-231613 - DOI: 10.25656/01:23161

https://nbn-resolving.org/urn:nbn:de:0111-pedocs-231613

https://doi.org/10.25656/01:23161

in Kooperation mit / in cooperation with:

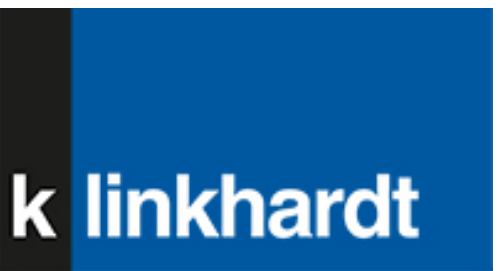

http://www.klinkhardt.de

\section{Nutzungsbedingungen}

Dieses Dokument steht unter folgender Creative Commons-Lizenz: http://creativecommons.org/licenses/by-nc-sa/4.0/deed.de - Sie dürfen das Werk bzw. den Inhalt unter folgenden Bedingungen vervielfältigen, verbreiten und öffentlich zugänglich machen sowie Abwandlungen und Bearbeitungen des Werkes bzw. Inhaltes anfertigen: Sie müssen den Namen des Autors/Rechteinhabers in der von ihm festgelegten Weise nennen. Diese Werk bzw. der Inhalt darf nicht für kommerzielle Zwecke verwendet werden. Die neu entstandenen Werke bzw. Inhalte dürfen nur unter Verwendung von Lizenzbedingungen weitergegeben werden, die mit denen dieses Lizenzbedingungen weitergegeben werden,
Lizenzvertrages identisch oder vergleichbar sind.

Mit der Verwendung dieses Dokuments erkennen Sie die

Nutzungsbedingungen an.

\section{Terms of use}

This document is published under following Creative Commons-License http://creativecommons.org/licenses/by-nc-sa/4.0/deed.en - You may copy, distribute and transmit, adapt or exhibit the work in the public and alter, transform or change this work as long as you attribute the work in the manner specified by the author or licensor. You are not allowed to make commercial use of the work. If you alter, transform, or change this work in any way, you may distribute the resulting work only under this or a comparable license.

By using this particular document, you accept the above-stated conditions of use.

\section{Kontakt / Contact:}

\section{peDOCS}

DIPF | Leibniz-Institut für Bildungsforschung und Bildungsinformation Informationszentrum (IZ) Bildung

E-Mail: pedocs@dipf.de

Internet: www.pedocs.de

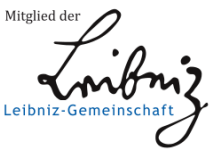




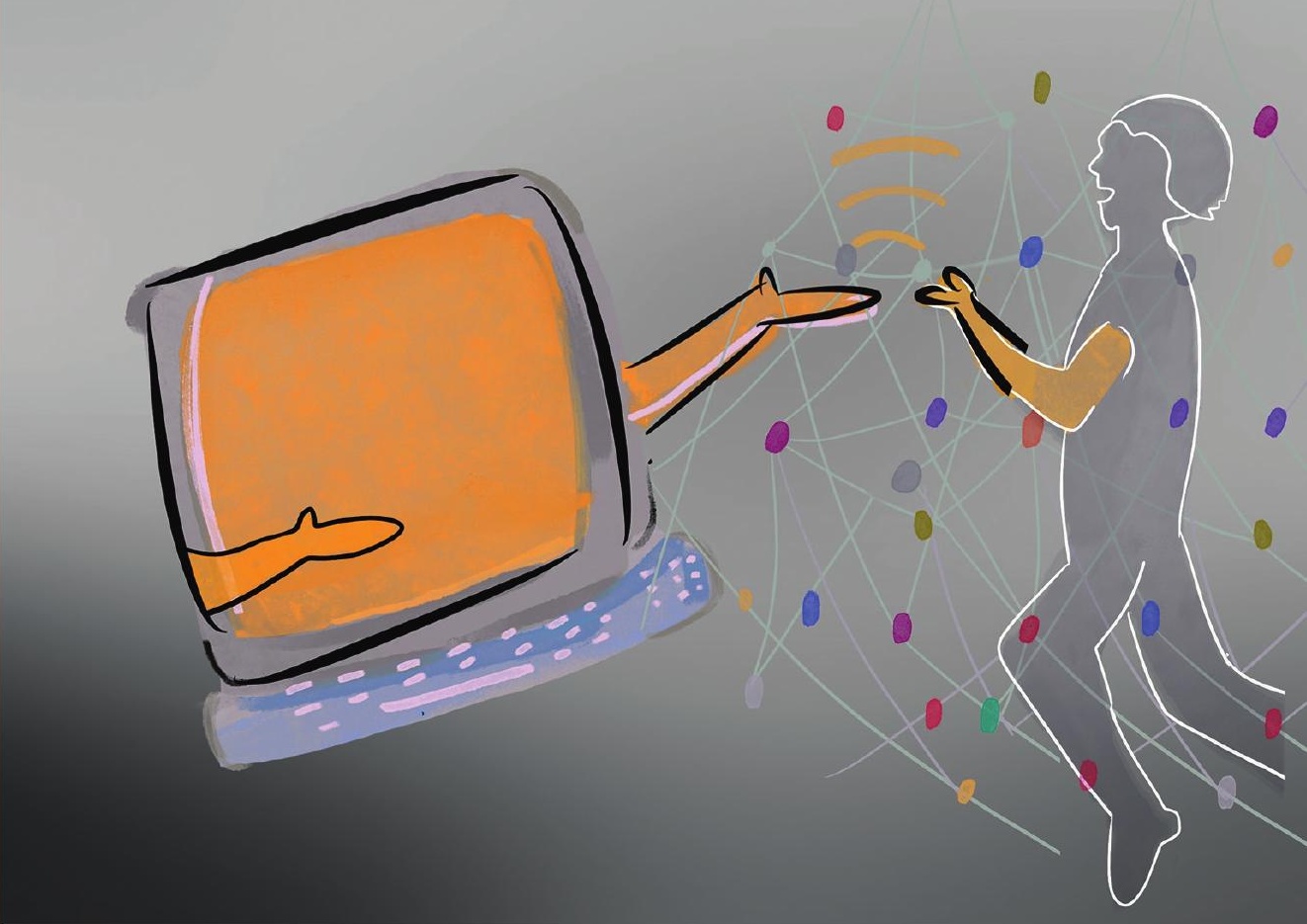

Maik Wunder (Hrsg.)

Digitalisierung und Soziale Arbeit

Transformationen und Herausforderungen 


\section{Maik Wunder \\ (Hrsg.)}

\section{Digitalisierung und Soziale Arbeit}

Transformationen und Herausforderungen 
Die Open Access-Publikation dieses Titels wurde durch Mittel des Forschungsschwerpunktes digitale_kultur der FernUniversität in Hagen finanziert.

Dieser Titel wurde in das Programm des Verlages mittels eines Peer-Review-Verfahrens aufgenommen. Für weitere Informationen siehe www.klinkhardt.de.

Bibliografische Information der Deutschen Nationalbibliothek

Die Deutsche Nationalbibliothek verzeichnet diese Publikation in der Deutschen Nationalbibliografie; detaillierte bibliografische Daten sind im Internet abrufbar über http://dnb.d-nb.de.

2021.h. () by Julius Klinkhardt.

Grafik Umschlagseite 1: (C) Miriam Tölgyesi (Rechte beim Herausgeber)

Druck und Bindung: AZ Druck und Datentechnik, Kempten.

Printed in Germany 2021.

Gedruckt auf chlorfrei gebleichtem alterungsbeständigem Papier.

Die Publikation (mit Ausnahme aller Fotos, Grafiken und Abbildungen) ist veröffentlicht unter der Creative Commons-Lizenz: CC BY-NC-SA 4.0 International https://creativecommons.org/licenses/by-nc-sa/4.0/

ISBN 978-3-7815-5911-0 digital doi.org/10.35468/5911

ISBN 978-3-7815-2473-6 print 


\title{
Inhaltsverzeichnis
}

\author{
Maik Wunder
}

Einleitung in den Band 9

\section{Digitalisierung und Veränderung Sozialer Arbeit}

\section{Udo Seelmeyer und Nadja Kutscher}

Zum Digitalisierungsdiskurs in der Sozialen Arbeit:

Befunde - Fragen - Perspektiven

\section{Maik Wunder}

Streiflichter durch Theorien zur Digitalisierung -

Digitalisierung als Gesellschafts-, Sozial- und Kulturtheorie

und deren mögliche Relevanz für die Soziale Arbeit

\section{Alexander Unger}

Digitalisierung oder Mediatisierung?

Ein analytischer Blick auf die Transformation

sozialpädagogischer Arbeitsfelder

Marc Witzel

Sozialpädagogische Orte im digitalen Raum

Philipp Waag

Digitalisierung als komplexer Gestaltungsspielraum:

Eine systemtheoretische Bestimmung disziplinärer und praktischer

Herausforderungen in der Sozialen Arbeit .80

\section{Digitalisierung und sozialarbeiterische Profession}

Fabian Hoose, Katrin Schneiders und Anna-Lena Schönauer

Von Robotern und Smartphones.

Stand und Akzeptanz der Digitalisierung im Sozialsektor

Anna-Sophie Brandt

Digitalisierung in der Gemeinwesenarbeit -

Bedarfe und Herausforderungen von Fachkräften in der Sozialen Arbeit 


\section{Diana Schneider}

Ein Schritt in Richtung De-Professionalisierung?

Plädoyer für eine intensive Diskussion über algorithmische Systeme in der professionellen Praxis

\section{Digitalisierung und Adressat*innen von Sozialer Arbeit}

Frieda Heinzelmann, Tanja Holzmeyer, Katrin Proschek und Frank Sowa

Digitalisierung als Projektionsfläche für Sehnsüchte und Ängste in Narrativen von wohnungslosen Menschen

Eva Maria Bäcker, Markus Grottke und Andreas König

Chancen digitaler Technologien für die Sozialen Arbeit?

Überlegungen zu Social Entrepreneurship unter Einsatz von digitalen

Lerntechnologien, virtuellem Kontext, New Work und

Selbstkontrollmechanismen

Anke Lang

Digitalisierung in der Kita - Bildung und Teilhabe für alle Kinder!

\section{Digitalisierung und sozialarbeiterische Ausbildung}

Martin Stummbaum und Kirsten Rusert

Zukünfte Sozialer Arbeit - digital und wie bei Ikea

Szenarien Sozialer Arbeit in der Digitalisierung

Joachim K. Rennstich

Neue Tricks für alte Hunde?

Digitalisierung als Herausforderung in Lehrvermittlung und Forschung

Jaqueline Veenker und Melanie Kubandt

Digitalisierung in Kindertagesstätten - Perspektiven von Lehrkräften an (Berufs-)Fachschulen für Sozialpädagogik 


\section{Digitalisierung und Forschung zu Sozialer Arbeit}

Angela Tillmann und André Weßel

Digitalisierung in der stationären Kinder- und Jugendhilfe zur Relevanz von digitalen Medien und Medienbildung in einem vernachlässigten Bildungskontext

Christian Ghanem, Markus Eckl, Robert Lehmann und Jean-Pierre Widerhold „Irgendwie fühle ich mich als Angehörige alleine gelassen“.

Eine automatisierte Analyse eines Onlineforums

für Angehörige von Inhaftierten

Almut Leh, Annabel Walz, Felix Engel und Matthias Hemmje

Historische Biografieforschung und Soziale Arbeit.

Interdisziplinäre Begegnungen im digitalen Raum .255 


\section{Sozialpädagogische Orte im digitalen Raum}

\section{Zusammenfassung}

Ausgehend von der theoriesystematischen Bestimmung von Sozialpädagogik als Ortshandeln diskutiert der vorliegende Beitrag zwei Aspekte digitaler Räume und deren Bedeutung für die sozialpädagogische Gestaltung von Räumen zu sozialpädagogischen Orten. Ziel ist es, einen Beitrag zu der Frage zu leisten, ob die mit digitalen Räumen einhergehenden Veränderungen der Qualität von Räumen für ein Verständnis von Sozialpädagogik als Ortshandeln bedeutsam sind. Dazu wird in einem ersten Schritt eine theoriesystematische Bestimmung sozialpädagogischen Handelns als Ortshandeln dargelegt. Diese wird mit neueren Diskussionen zu sozialen und insbesondere digitalen Räumen verknüpft. Anhand von zwei Merkmalen digitaler Räume wird dann die Frage nach der Bedeutung für sozialpädagogisches Ortshandeln gestellt.

Die Versuche, Räume - insbesondere soziale Räume - definitorisch zu fassen, sind vielseitig und reichen von der Konzeptionierung als Containerräume bis zu relationalen Raumkonzepten, die das Raumverständnis entlang sozialer Beziehungen des einzelnen Individuums fassen. In der aktuellen Diskussion werden Räume in erster Linie als relational konzipiert (Masson 2016, 100ff.).

Eine solche Konzeptionierung des Raumes ist in sozialpädagogischen Diskursen u. a. bereits für die Jugendarbeit theoretisch entwickelt und diskutiert worden. Allerdings beziehen sich diese Überlegungen zumeist auf eine vorhandene materielle Dimension des Raumes. Ähnliches gilt für die Auseinandersetzungen um sozialraumorientierte Soziale Arbeit (vgl. Deinet 2009, 2010; Lingg \& Stiehler 2010, 185).

Erweitert wird diese Perspektive durch die Diskurse um digitale virtuelle Räume, welche nicht notwendigerweise eine materiell-physische Entsprechung aufweisen müssen. Diese können als eine spezifische Form von Raum betrachtet werden, der die Konzeption von Relationalität von der materiellen Dimension abstrahiert ${ }^{1}$ und in diesem Prozess radikalisiert. Der vorliegende Beitrag fragt nach der Be-

1 In der Abstraktion wird der digitale virtuelle Raum scheinbar von der Materialität der Hardware getrennt, die dennoch die Grundlage bleibt und damit auch Teil des Raums ist. 
deutung dieser Veränderung der Qualität von Räumen für ein Verständnis von Sozialpädagogik als „Ortshandeln“ (Winkler 1999).

Die hier vorgelegten Überlegungen haben den Charakter offener Überlegungen und stellen somit keine abgeschlossene theoretische Figur dar - die Hinweise und Diskussionen im Entstehungsprozess des Beitrags ${ }^{2}$ machen stattdessen die Notwendigkeit einer weiteren und zukünftigen Auseinandersetzung mit dem Thema deutlich. In Form von Analogien und Differenzen werden zwei Aspekte im Verhältnis von Sozialpädagogik und digitalen Räumen diskutiert. Ausgehend von dem theoriesystematischen Bestimmungsversuch, Soziale Arbeit als Ortshandeln zu fassen, den Ort also als einen der zentralen theoretischen Reflexionsbegriffe für ein Verständnis von Sozialpädagogik zu thematisieren, gilt die formulierte Frage der Aktualität dieses Konzeptes einerseits und der Anschlussfähigkeit und Bedeutung eines solches Verständnisses sozialpädagogischen Handelns an aktuelle Entwicklungen andererseits. Die Bestimmung von Sozialpädagogik als Ortshandeln wird $1988^{3}$ von Michael Winkler in seinem Theorieentwurf „Eine Theorie der Sozialpädagogik" vorgenommen und ist historisch vor den Diskursen um virtuelle und digitale Räume verortet. Dennoch wird dieser theoriesystematische Bestimmungsversuch auch weiterhin als bedeutsam für die sozialpädagogische Theoriediskussion gekennzeichnet. In ihm werden die Begriffe Subjekt und Ort als die zentralen Reflexionsbegriffe sozialpädagogischen Handelns miteinander in Bezug gesetzt. Insbesondere vor dem Hintergrund der sich verändernden Konzeptionen und Grundlagen eines Raum- und Ortsverständnisses ist die Diskussion um das Verhältnis des sozialpädagogischen Ortes zum digitalen Raum notwendig, um einen theoretisch informierten Beitrag zur Rolle und Bedeutung sozialpädagogischen Handelns in virtuellen Räumen zu leisten.

In einem ersten Schritt werden die für die hier dargelegten Überlegungen bedeutsamen Aspekte des „sozialpädagogischen Ortes 4“ skizziert und deren Anschlussfähigkeit an relationale Raumkonzepte verdeutlicht. In einem zweiten Schritt wird dann das Verständnis digitaler Räume erläutert, welches hier zugrunde gelegt wird und dieses mit den vorausgegangenen Überlegungen diskutiert. Anhand von zwei bedeutsamen Unterschieden zwischen digitalen und nichtdigitalen Räumen werden beispielhaft neue Aspekte für sozialpädagogisches Handeln als Ortshan-

2 Für Hinweise und Kommentare danke ich den Teilnehmer*innen der Tagung „Digitalisierung und Soziale Arbeit - Transformationen, Beharrungen und Herausforderungen“ am 22. \& 23.10.2020 sowie Maik Wunder als Herausgeber dieses Bandes.

3 Die Neuauflage dieses Theorieentwurfs nach über 30 Jahren weist auf die aktuelle Bedeutung des Werkes hin (Winkler 2021).

4 Der Begriff des sozialpädagogischen Ortes als feststehender theoretischer Begriff wird im Sinne von sozialpädagogischer Raum genutzt. Die Ausführungen Winklers weisen darauf hin, dass eine analytische Differenzierung zwischen Raum und Ort im Sinne der raumsoziologischen Begriffsdifferenzierung nicht sinnvoll scheint (vgl. Winkler 1988, 297ff.). 
deln problematisiert. Anhand dieser beiden Aspekte soll ein erster Versuch einer Diskussion unternommen werden, ob die hier thematisierte Perspektive auch im Rahmen von Digitalisierungsprozessen noch ihren umfassenden theoretischen Erklärungsanspruch aufrechterhalten kann - sie also trotz gesellschaftlichen Veränderungen weiterhin herangezogen werden kann, um Aussagen über die Sozialpädagogik zu treffen.

\section{Der sozialpädagogische Ort}

Sozialpädagogisches Handeln als Ortshandeln zu bestimmen verweist auf die zentrale Stellung des Subjekts als Bedingung des sozialpädagogischen Handelns. Das Subjekt ist u.a. dadurch gekennzeichnet, dass es seine Subjektivität nicht aufgeben kann (vgl. Winkler 1988, 267ff.). Mit dieser Bedingung geht einher, dass Subjekte „noch in der Aufgabe von Autonomie“ (Winkler 2013, 152) als selbsttätige Subjekte verstanden werden müssen. Allerdings können sie eine Erfahrung machen, die Winkler als Modus der Differenz bezeichnet. Das Subjekt im Modus der Differenz bezeichnet einen Zustand des Subjekts, in dem ,wir uns mit uns selbst uneins fühlen, dabei nicht so recht wissen, wo wir stehen, wie wir uns verhalten sollen. Uns ist dann der Zusammenhang zwischen unserem Tun und der realen Welt entglitten" (Winkler 1988, 153). Der Modus der Differenz resultiert aus dem Scheitern von Aneignungsprozessen und weist eine Alltäglichkeit auf, die diesen erst einmal banal wirken lässt. Allerdings wird selbiger zu einem Krisenzustand, wenn das Subjekt keine Kontrolle mehr über seine Lebensbedingungen hat. Wenn „sich das Subjekt einer ihm fremden, äußerlichen Kontrolle unterworfen“ (Winkler 1988, 153) sieht und sich im „Subjektmodus der aufgehobenen Subjektivität“ (ebd., 153) befindet.

Um dem Subjekt zu ermöglichen, den Modus der Identität zu erreichen und also die Bedingungen seines Lebens zu kontrollieren, indem das „Subjekt an der gesellschaftlichen Praxis teilnimmt und so den dort vollzogenen Beurteilungsverfahren unterworfen ist" (ebd., 333), müssen Aneignungsprozesse gelingen. Um den krisenhaft gewordenen Zustand des Modus der Differenz zu überwinden, stellt sozialpädagogisches Handeln in Form des Ortshandelns den Subjekten Aneignungsmöglichkeiten zur Verfügung. Sozialpädagogisches Handeln meint Ortshandeln im Sinne der Gestaltung eines Ortes, welcher dann dem Subjekt im Modus der Differenz zur Aneignung zur Verfügung gestellt wird. „Indem das sozialpädagogische Handeln sich in der Gestalt des Ortes vergegenständlicht, schafft es die Voraussetzung für einen Bildungsprozeß des Subjekts“ (ebd., 277). Der sozialpädagogische Ort soll es den Subjekten ermöglichen, sich diesen anzueignen und durch den gelingenden Aneignungsprozess ihre eigene Subjektivität zu erfahren, 
handlungsfähig zu werden und den Modus der Differenz zu überwinden. Der sozialpädagogische Ort bemisst sich dementsprechend daran, ob es dem Subjekt im Modus der Differenz möglich ist ihn sich anzueignen.

Sozialpädagogische Orte bedürfen der Gestaltung durch Sozialpädagog*innen, sie sind nur als gestaltete und gestaltbare Orte denkbar. Damit schließt eine solche Vorstellung von Raum an aktuelle raumsoziologische Überlegungen an, die Raum nicht ontologisch fassen, sondern als einen sozialen Raum begreifen. Der Raum ist nicht natürlich da, sondern wird sozial hergestellt.

\section{Soziale Räume}

Räume, so Löw und Sturm, seien zu verstehen „als (An)Ordnungen von Lebewesen und sozialen Gütern an Orten “ (Löw \& Sturm 2019, 15). Als solche stellen Räume immer zugleich eine (gesellschaftliche) Struktur wie auch konkretes Handeln dar. Das Handeln realisiert sich dabei sowohl in der Praxis des Platzierens wie auch in der Relationierung und Zusammenfassung von sozialen Gütern und Lebewesen zu Räumen (ebd., 15).

Der Prozess der Herstellung des Raumes basiert dann auf zwei „sich in der Regel gegenseitig bedingenden Prozessen: der Syntheseleistung und dem Spacing (ebd., 17). Die Syntheseleistung besteht in der aktiven Verknüpfung der unterschiedlichen Elemente durch Menschen, die soziale Güter und Lebewesen zu Räumen zusammenfassen. Der Prozess des Spacing dagegen meint die Positionierung von sozialen Gütern und Lebewesen. Wesentlich ist, dass Spacing sowohl „den Moment der Platzierung als auch die Bewegung zur nächsten Platzierung" (ebd., 17) meint. In dieser doppelten Bedeutung von Platzierung werden der Prozess der Raumkonstitution und die Dynamik von Räumen noch einmal besonders hervorgehoben. Die Prozesse des Spacing und der Syntheseleistung werden dabei nicht von einzelnen Subjekten vollzogen, sondern in wechselseitigen Handlungen von Subjekten, die als solche immer selbst sozial sind (vgl. Löw 2020, 152). Beide Prozesse zeichnen sich durch eine Gleichzeitigkeit aus, da Prozesse des Spacing ohne Syntheseleistung nicht möglich sind.

Ein solches Verständnis begreift jeden Raum als sozialen Raum. Es widerspricht der Vorstellung von Containerräumen, die dem Sozialen vorgängig seien und durch das Handeln der Akteure erst gefüllt würden oder die gänzlich unabhängig von der sozialen Gestaltung existieren. Ob ein bestimmtes Gebäude ein Jugendzentrum, eine stationäre Wohngruppe, eine privat genutzte Wohnimmobilie oder etwas völlig anderes ist, wird nicht durch das Gebäude selbst vorgegeben. Erst durch eine spezifische Form der Gestaltung und Aneignung wird der Raum bestimmt (vgl. Löw \& Sturm 2019, 17). Raum wird als Sozialraum begriffen, der 


\section{Marc Witzel}

durch die Subjekte, ihr Verhältnis zueinander und zu sozialen Gütern im Raum bestimmt wird. Soziale Güter bezeichnet dabei sowohl die mit Bedeutung aufgeladenen Objekte im Raum wie auch die gültigen Regeln und Symbolsysteme. Eine solche Definition begreift Raum als konstruierte und damit auch als eine prinzipiell konstruierbare Struktur, die immer wieder auch dem Gestalten von Raum selbst vorgängig ist. Räume entstehen durch das Zusammenfassen von Menschen und sozialen Gütern in Wahrnehmungs-, Vorstellungs- oder Erinnerungsprozessen - der Synthese zu einem Raum -, die wiederum die Grundlage der Platzierung von Menschen und sozialen Gütern bilden, der (An-)Ordnung (vgl. ebd., 17).

\section{Virtuelle und digitale Räume}

Das Reden von Räumen thematisiert häufig implizit ihre materiell-physische Entsprechung in Form von Orten, Gebäuden oder Territorien. Die Rede von virtuellen Räumen dagegen weist auf das scheinbare Fehlen einer materiell-physischen Entsprechung hin, indem diese häufig als nicht körperlich erfahrbar und ohne territoriale Gebundenheit beschrieben werden (vgl. Tillmann 2010, 149; Hipfl 2004, 25). Virtuelle Räume entstehen durch Kommunikationsakte, in welchen die Syntheseleistungen und Platzierungsprozesse stattfinden. Da dies scheinbar ${ }^{5}$ unabhängig von territorialer Gebundenheit und materiell-physischen Entsprechungen der Räume geschieht, wird die Radikalität des oben skizzierten Raumverständnisses noch einmal deutlich, da die grundsätzlichen Ausführungen zum relationalen Raumbegriff ebenso für virtuelle Räume gelten. Räume entstehen somit ohne eine Verortung, ohne ein benennbares territoriales Gegenstück, sondern durch eine Syntheseleistung und die (An-)Ordnung von Menschen und sozialen Gütern in ihnen in kommunikativen Prozessen ${ }^{6}$.

Virtuelle Räume sind allerdings nicht zwingend auf digitale Techniken angewiesen, sondern realisieren sich beispielsweise in Telefonaten oder im Austausch von Briefen. Im Rahmen der Kommunikation finden sowohl Platzierungsprozesse statt wie auch die Synthese zu einem gemeinsamen Raum vollzogen wird. Durch die fortschreitenden Mediatisierungs- und Digitalisierungsprozesse nimmt die

5 Das auch in virtuellen Räumen keineswegs jeder sein kann, wer er mag, zeigen die unterschiedlichen Studien zur digitalen Ungleichheit, in denen die Rückbindung an die jeweilige soziale Situation der Subjekte sich immer wieder als bedeutsam für die konkrete Kommunikation mittels digitaler Medien erweist (vgl. Klein 2007; Zillien 2009). Der eigene soziale Standort, die gesellschaftliche Verortung der Individuen scheint durchaus bedeutsam zu bleiben.

6 Dabei werden die materiellen Grundlagen der virtuellen Räume ausgeblendet. Die Grundlagen des Virtuellen und des Handelns in virtuellen Räumen stellen als Teil der (An-)Ordnungen sozialer Güter auch technische Artefakte und Technologien in konkreter materieller Form dar. 
Verbreitung virtueller Räume auf Basis digitaler Kommunikation jedoch weiter zu. Digitale Räume sind als eine spezifische Form virtueller Räume zu verstehen, die sich dadurch auszeichnet, dass die ihnen zugrunde liegende Kommunikation - die sozialen Gestaltungsprozesse der Räume mittels Platzierung und Syntheseleistungen - digital erfolgt. An die Stelle einer materiell-physischen Entsprechung tritt dann eine hard- und softwarebasierte ${ }^{7}$ Strukturierung dieser Räume (vgl. Unger 2010, 100). Eine solche hard- und softwarebasierte Strukturierung digitaler Räume präsentiert sich den Subjekten als eine Struktur, welche bereits eine (An) Ordnung mit sich bringt. Diese kann sich als eher starr und standardisiert präsentieren oder auch als gestaltbar und mit vielfältigen Optionen. Diese Differenzierung macht danah boyd (2011) anhand der Gestaltungsmöglichkeiten und der damit einhergehenden Ästhetik zwischen den Sozialen Online-Netzwerken MySpace und Facebook aus und beschreibt zugleich damit einhergehende Prozesse der Raumkonstitution (ebd. 2011, 212ff.). Durch die Gestaltung der jeweiligen Online-Profile und der Vernetzung mit anderen Nutzern finden Platzierungsprozesse ebenso statt, wie Syntheseleistungen, in denen die durch die Strukturierung bereits vorhandene (An)Ordnung sozialer Güter und Menschen einen Raum darstellt, der den Subjekten zur Aneignung gegenübersteht.

Digitale Räume ähneln also in ihren Konstitutionsprozessen durchaus nichtdigitalen Räumen. Zudem weisen sie in Analogie zu den materiell-physischen Entsprechungen nichtdigitaler Räume sowohl eine vorhandene Struktur wie auch eine Grenze auf. Sie ähneln nichtdigitalen Räumen darin, dass auch in ihnen (An) Ordnungsprozesse stattfinden und sie eine grundlegende Struktur und Begrenzung aufweisen, anhand derer ein Wechsel zwischen unterschiedlichen Sozialen Online-Netzwerken sicht- und erfahrbar wird, so dass der Wechsel zwischen den digitalen Räumen den Subjekten durchaus deutlich ist.

Zugleich unterscheiden sich digitale Räume von nichtvirtuellen Räumen darin, dass sie die (An)Ordnung der Subjekte im Raum nicht offensichtlich und leiblich erfahrbar machen. Wer sich wann mit wem innerhalb digitaler Räume bewegt wie also die Struktur des digitalen Raumes zu einem bestimmten Zeitpunkt von den Subjekten wahrgenommen wird - bleibt je nach softwarebasierter Gestaltung des Raums mehr oder weniger unklar. Damit wird die Syntheseleistung ebenso wie der Platzierungsprozess noch einmal deutlich expliziter zu einer Aufgabe der Subjekte. Dies bedeutet für sozialpädagogische Kontexte, dass den Räumen und deren Gestaltung eine ganz wesentliche Bedeutung zukommt, da sowohl Erzieher wie auch Zöglingssubjekt sich unter Umständen in digitalen Räumen nicht, nicht wissentlich oder ganz ungewollt begegnen.

7 Dies umfasst die Hardware der Nutzer*innen ebenso wie die der Anbieter*innen und Service-Provider. 


\section{Herausforderungen digitaler Räume für sozialpädagogisches Ortshandeln}

Aufgrund der Differenz von digitalen Räumen und anderen sozialen Räumen ist davon auszugehen, dass digitale und nichtdigitale Räume von den Subjekten unterschiedlich erfahren werden. Für die Frage, ob und wie sozialpädagogische Orte sich im digitalen Raum realisieren lassen, sind mindestens zwei Aspekte bedeutsam. Zum einen die binäre Differenz von Teilhabe an einem digitalen Raum und zum anderen die potenziell dauerhafte Präsenz der digitalen Räume.

\section{Die binäre Differenz von in und out}

Digitale Räume und deren Grenzen ermöglichen kaum fließende Übergänge. Die Teilhabe an einem digitalen Raum lässt sich in die binäre Differenz von drinnen und draußen, von in und out differenzieren. Die Konstitution nichtvirtueller Räume dagegen ist an materiell-physische Entsprechungen des Raumes geknüpft und weist in der Regel einen Ortsbezug auf. Ein Ort ist zunächst „eine Stelle, konkret benennbar, meist geographisch benennbar" (Löw 2020, 156), welche „raumkonstituierenden Elementen die Möglichkeit [bietet, M.W.], dort gelagert zu werden" (ebd., 156). Diese Orte und materiell-physischen Entsprechungen von Räumen sind in Verbindung mit der Bewegung der Subjekte in den Bahnenräumen (vgl. Löw 2020, 156ff.) eingebunden in ständige Platzierungsprozesse der Subjekte. Raumkonstitution vollzieht sich als Prozess in der Gleichzeitigkeit von Platzierung und Syntheseleistung und mit der Bewegung des Subjekts zwischen zwei Räumen oder Platzierungen wiederholen sich diese Prozesse immer wieder. In jedem neuen Platzierungsprozess und jeder neuen Syntheseleistung kann eine Annäherung an einen Ort einerseits und eine Platzierung in der Peripherie eines Raums stattfinden. Diese Prozesse ermöglichen eine Annäherung an Orte und Räume und damit jeweils die Aneignung des Ergebnisses der vorausgegangenen Platzierung und Syntheseleistung.

Digitale Räume bieten solche Annäherungsprozesse kaum. Entweder man ist in den jeweiligen digitalen Raum eingetreten oder man ist es nicht. Bereits Ende der 1990er Jahre wurde der nicht vorhandene Prozess der Annäherung mit der überraschten Frage „Bin ich da schon drin, oder was? “8 in der Werbung eines großen Internetanbieters verdeutlicht. Einen Blick auf den Raum von außerhalb, ein langsames und vorsichtiges Herantasten an den Raum ist in digitalen Räumen nur bedingt möglich. Eine WhatsApp-Gruppe lässt sich nicht aus der Distanz beobachten. Dagegen ermöglichen es Räume, die über eine materiell-physische Entsprechung verfügen, sich ihnen anzunähern und damit in der Annäherung bereits mit der Aneignung des Ortes zu beginnen. Dies ermöglicht es, der Bedro-

8 https://www.youtube.com/watch?v=4XIwVMYeP4I 
hung der Subjektivität durch den Ort schrittweise durch gelingende Aneignungsprozesse zu begegnen. Ähnlich dem Scheinriesen aus „Jim Knopf und Lukas der Lokomotivführer" verliert der Ort an Bedrohlichkeit durch einen schrittweisen Annäherungs- und Aneignungsprozess (vgl. Ende 1990, 124ff.).

Für sozialpädagogische Orte sind diese Annäherungen als Teil der Aneignungsprozesse in dem Begriff des „Oszillieren zwischen der Innenwelt des Ortes und der gesellschaftlichen Außenwelt" (Winkler 1988, 299) beschrieben. Gerade dort, wo Aneignungsprozesse zu scheitern drohen, ist die Möglichkeit des Rückzugs und einer erneuten Annäherung, der Wechsel zu einer bekannten oder weniger die Subjektivität bedrohenden Platzierungskonstellation die Grundlage für den Erhalt des Modus der Identität des Subjekts. Die Gestaltung des sozialpädagogischen Ortes im digitalen Raum steht vor der Herausforderung, diesen so zu gestalten und immer wieder anzupassen, dass er im digitalen Raum schrittweise Aneignungsprozesse ermöglicht, die das Subjekt nicht überfordern.

\section{Die dauerhafte Präsenz digitaler Räume}

Die binäre Differenzierung zwischen in und out geht dabei einher mit einer zweiten Herausforderung der sozialpädagogischen Gestaltung digitaler Räume - deren dauerhafter Präsenz.

Räume mit einer materiell-physischen Entsprechung bieten die Möglichkeit, diese zeitweise zu verlassen oder sie sogar gänzlich hinter sich zu lassen. Darin liegt ein Moment der Entlastung von der Aneignungsaufgabe und eine Möglichkeit, mit dem Scheitern von Aneignungsprozessen umzugehen. Eine Ortsveränderung ermöglicht dem Subjekt einen neuen Ort anzueignen oder den Aneignungsprozess zu unterbrechen (vgl. ebd., 297; Winkler 2009). Sozialpädagogische Leistungen zeichnen sich zum Teil durch eine solche Ortsveränderung aus. So können die stationären Erziehungshilfen fast prototypisch als eine Ortsveränderung gelten, die das Subjekt von den Orten entfernt, die seine Subjektivität schädigen und die das Subjekt aus eigener Kraft nicht mehr verlassen kann (vgl. Trede $\&$ Winkler 2006).

Digitale Räume dagegen konstituieren sich vor allem durch kommunikative Akte, begleiten den Wechsel des materiell-physischen Ortes selbst und bleiben unabhängig von der territorialen Verortung des Subjekts als sozialer Raum bestehen. Sie können somit dauerhaft präsent sein. Für die Gestaltung sozialpädagogischer Orte gilt es, die Möglichkeit der dauerhaften Präsenz digitaler Räume zu bedenken. Die dauerhafte Präsenz digitaler Räume ist dabei in zwei Dimensionen bedeutsam.

Erstens wirkt sie in den materiell-physischen sozialpädagogischen Ort hinein, so dass an diesem ein Bruch mit den bisherigen Lebenswelten nicht mehr in dem Maße und der Radikalität vollzogen werden kann, wie es historisch beispielsweise die Anstaltserziehung tat (vgl. Witzel 2015, 125). Diesen Bruch dort herzustel- 
len, wo das Subjekt nicht mehr selbst dazu in der Lage ist über seine Lebensbedingungen zu verfügen, wird dann erschwert, wenn sich die Bedingungen des Modus der Differenz auch aus der Teilhabe an digitalen Räumen ergeben. Der sozialpädagogische Ort kann dann unter Umständen seine Aufgabe nicht mehr erfüllen, nämlich dem Subjekt Bedingungen zur schaffen, unter denen es seine Subjektivität erfahren kann.

Zweitens kann die dauerhafte Präsenz digitaler Räume dann problematisch werden, wenn der sozialpädagogische Ort im digitalen Raum realisiert wurde. Gelingt es dem Subjekt nicht, den sozialpädagogischen Ort wieder zu verlassen, ihn im Prozess der Aneignung obsolet zu machen, kann gerade dadurch das Scheitern des Aneignungsprozesses begründet sein (vgl. Winkler 1988, 280f.). Auch hier ist es notwendig, den sozialpädagogischen Ort immer wieder neu zu gestalten und anzupassen und zugleich dem Subjekt die Möglichkeit zu geben, den Ort zu überwinden.

Die grundsätzliche Relevanz digitaler Räume in den Lebenswelten der Subjekte macht jedoch deutlich, dass diese auch in sozialpädagogischen Kontexten nicht zu ignorieren sind. Digitale Räume müssen grundsätzlich in sozialpädagogischen Handlungszusammenhängen berücksichtigt werden. Die dauerhafte Präsenz des digitalen Raums wird so zu einer Herausforderung für die Gestaltung des sozialpädagogischen Ortes. Denn eine Gestaltung und eine Überprüfung hinsichtlich ihrer Potenziale, dem Subjekt gelingende Aneignungsprozesse zu ermöglichen, erfordern nicht nur die materiell-physisch erfahrbare Räume, sondern auch die digitalen Räume. Sozialpädagogisches Ortshandeln enthält somit eine Dimension im digitalen Raum.

\section{Anstelle eines Schlusses}

Die dargelegten Überlegungen lassen keine abschließenden Schlüsse zu, welche Bedeutung der qualitativen Veränderung des Raums für ein Verständnis von Sozialpädagogik als Ortshandeln zukommt. Stattdessen weisen sie auf die besonderen Herausforderungen hin, die einem solchen Verständnis von Sozialpädagogik mit Blick auf digitale Räume gegenüberstehen. Keineswegs scheinen die Überlegungen zum sozialpädagogischen Ort durch die Entstehung digitaler Räume obsolet zu werden. Allerdings weisen die Überlegungen vor dem Hintergrund der Relevanz digitaler Räume in den Lebens- und Alltagswelten der Menschen auf die Notwendigkeit, sich mit diesen in Prozessen sozialpädagogischen Ortshandelns auseinanderzusetzen.

Keineswegs ausreichend ist dann eine Reduzierung der sozialpädagogischen Auseinandersetzung auf Online-Beratungsangebote als Ressource oder Soziale On- 
line-Netzwerke als Risiken des Handelns. Vielmehr muss die Interdependenz zwischen digitalen Räumen und den anderen Aspekten der Lebenswelten unter den Bedingungen von Mediatisierungsprozessen in den Blick genommen werden. Digitale Räume sind Teil der Lebenswelten.

Begreift man sozialpädagogisches Handeln als Ortshandeln, spielt die Gestaltung des Ortes eine herausragende Bedeutung. Gerade die beiden benannten Dimensionen digitaler Räume machen deutlich, dass diese eine veränderte Qualität von Räumen beschreiben. Die am Beispiel der binären Differenz von in und out und der potenziell dauerhaften Präsenz digitaler Räume beschriebene Differenz zu nicht-digitalen Räumen verweist auf eine andere Erfahrung, ein anderes Erleben des Raumes und seiner Grenzen.

Digitale Räume erfordern damit eine Thematisierung in ihrem jeweiligen Verhältnis zu den Bedingungen der Subjektivität. Weder kann sozialpädagogisches Ortshandeln digitale Orte als Teil der Lebenswelten ignorieren noch diese ausschließlich als sozialpädagogische Orte konstruieren, die unabhängig von digitalen Räumen seien. Vielmehr stellen digitale Räume bereits eine gesellschaftliche Realität dar, die auch für die sozialpädagogische Gestaltung von Orten von Bedeutung ist. Dies kann bedeuten die digitalen Räume nicht an sozialpädagogische Orte vordringen zu lassen, welche die Subjektivität schädigen; dort wo es für das Bestehen der Subjekte notwendig ist einen Bruch mit dem digitalen Ort zu vollziehen. Zugleich gilt es die Subjekte in der Aneignung der digitalen Räume zu unterstützen, die ihnen ermöglichen den Modus der Differenz zu überwinden. Die Vielzahl der für das Individuum bedeutsamen digitalen Räume grundsätzlich im pädagogischen Ortshandeln mitzudenken stellt eine hohe Anforderung dar. Sozialpädagogisches Ortshandeln in digitalen Räumen muss deren Eigenheiten bedenken und differenzieren. Für digitale Räume lassen sich zwei mögliche Handlungsansätze differenzieren, wie digitale Räume zu sozialpädagogischen Orten werden können.

Zum einen besteht die Möglichkeit, in bereits bestehenden digitalen Räumen gestaltend zu agieren und die (An)Ordnung der Menschen und sozialen Güter zu verändern, also Syntheseleistungen und Platzierungsprozesse zu initiieren. Ein solches Vorgehen läuft allerdings immer Gefahr, Grenzen zu überschreiten oder zu Entgrenzungsprozessen zu führen. Wo Sozialpädagog*innen in WhatsApp-Familiengruppen Mitglied werden, besteht keine Möglichkeit des Rückzugs mehr - weder für die Familie noch für die Professionellen.

Als weitere Option können sozialpädagogische Orte als eigener digitaler Raum geschaffen werden. Digitale Kommunikationsmöglichkeiten in stationären Einrichtungen der Jugendhilfe oder Online-Beratungsangebote können hier konkrete Beispiele sein. Diese existieren in der Regel aber nur bedingt und vor allen Dingen nicht ausschließlich in den Lebenswelten der Subjekte. Diese Angebote können 
andere Soziale Online-Netzwerke und Kommunikationszusammenhänge nicht ersetzen, sondern lediglich ergänzen.

Während sozialpädagogisches Ortshandeln in nichtdigitalen Räumen inzwischen stark ausdifferenziert ist und damit eine große Breite an sozialpädagogischen Orten geschaffen hat, bleibt das digitale Ortshandeln noch auf eher wenige Bereiche bezogen. Dass es einer vermehrten Auseinandersetzung auch mit digitalen Orten in Disziplin und Profession bedarf, scheint unter dem hier zugrunde gelegten theoretischen Zugang offensichtlich. Eine solche Auseinandersetzung ist einerseits die Grundlage, um digitale Räume sozialpädagogisch zu gestalten, andererseits Bedingung eines umfassenden Verständnisses der Lebens- und Umwelten der Subjekte.

Für eine theoriesystematische Auseinandersetzung um die Frage der Bedeutung digitaler Räume für sozialpädagogisches Ortshandeln scheint insbesondere eine Auseinandersetzung mit der von Winkler entwickelten Typologie sozialpädagogischer Orte in zukünftigen Diskussionen gewinnbringend zu sein (vgl. Winkler 1988, 297ff.). Insbesondere Fragen zur räumlichen und zeitlichen Entgrenzung sowie zu den Zugängen und Möglichkeiten der Überwindung sozialpädagogischer Orte scheinen für diese Diskussionen bedeutsam.

\section{Quellenangaben}

boyd, danah (2011): White Flight in Networked Publics: How Race and Class Shaped American Teen Engagement with MySpace and Facebook. In: Nakamura, Lisa \& Chow-White, Peter (Hrsg.): Race after the Internet. New York, NY: Routledge, 203-222.

Deinet, Ulrich (2009): „Aneignung“ als Bildungskonzept sozialräumlicher Jugendarbeit. In: Deinet, Ulrich (Hrsg.): Sozialräumliche Jugendarbeit. Wiesbaden: VS Verlag für Sozialwissenschaften, 143160.

Deinet, Ulrich (2010): Aneignung öffentlicher und virtueller Räume durch Jugendliche. In: Cleppien, Georg \& Lerche, Ulrike (Hrsg.): Soziale Arbeit und Medien. Wiesbaden: VS Verlag für Sozialwissenschaften, 37-51.

Ende, Michael (1990): Jim Knopf und Lukas der Lokomotivführer. Stuttgart \& Wien: Thienemann. Hipfl, Brigitte (2004): Mediale Identitätsräume. Skizzen zu einem ,spatial turn` in der Medien- und Kommunikationswissenschaft. In: Hipfl, Brigitte; Klaus, Elisabeth \& Scheer, Uta (Hrsg.): Identitätsräume. Bielefeld: transcript Verlag, 16-50.

Klein, Alexandra (2007): Soziales Kapital online: Soziale Unterstützung im Internet. Eine Rekonstruktion virtualisierter Formen sozialer Ungleichheit. Bielefeld: Universität Bielefeld. Online unter: http://nbn-resolving.de/urn:nbn:de:hbz:361-12601 (Abrufdatum: 22.08.2011).

Lingg, Eva \& Stiehler, Steve (2010): Ort. In: Reutlinger, Christian; Fritsche, Caroline \& Lingg, Eva (Hrsg.): Raumwissenschaftliche Basics. Wiesbaden: VS Verlag für Sozialwissenschaften, 181-190.

Löw, Martin (2020): In welchen Räumen leben wir? Eine raumsoziologisch und kommunikativ konstruktivistische Bestimmung der Raumfiguren Territorialraum, Bahnenraum, Netzwerkraum und Ort. In: Reichertz, Jo (Hrsg.): Grenzen der Kommunikation - Kommunikation an den Grenzen. Weilerswist: Velbrück Wissenschaft, 149-164.

Löw, Martina \& Sturm, Gabriele (2019): Raumsoziologie: Eine disziplinäre Positionierung zum Sozialraum. In: Kessl, Fabian \& Reutlinger, Christian (Hrsg.): Handbuch Sozialraum. Wiesbaden: Springer Fachmedien Wiesbaden, 3-21. 
Masson, Silke (2016): Die Mittelschicht in benachteiligten Stadtteilen. Wiesbaden: Springer Fachmedien Wiesbaden.

Tillmann, Angela (2010): Medienwelt. In: Reutlinger, Christian; Fritsche, Caroline \& Lingg, Eva (Hrsg.): Raumwissenschaftliche Basics. Wiesbaden: VS Verlag für Sozialwissenschaften, 149-157.

Trede, Wolfgang \& Winkler, Michael (2006): Stationäre Erziehungshilfen: Heim, Wohngruppe, Pflegefamilie. In: Krüger, Heinz-Hermann \& Rauschenbach, Thomas (Hrsg.): Einführung in die Arbeitsfelder des Bildungs- und Sozialwesens. Opladen [u.a.]: Budrich, 251-266.

Unger, Alexander (2010): Virtuelle Räume und die Hybridisierung der Alltagswelt. In: Grell, Petra; Marotzki, Winfried \& Schelhowe, Heidi (Hrsg.): Neue digitale Kultur- und Bildungsräume. Wiesbaden: VS Verlag für Sozialwissenschaften, 99-117.

Winkler, Michael (1988): Eine Theorie der Sozialpädagogik. Stuttgart: Klett-Cotta.

Winkler, M. (1999): Ortshandeln: Die Pädagogik der Heimerziehung. In: Colla, H. E. (Hrsg.): Handbuch Heimerziehung und Pflegekinderwesen in Europa. Neuwied: Luchterhand, 307-323.

Winkler, Michael (2009): Der pädagogische Ort. In: Macha, Hildegard; Witzke, Monika; Ladenthin, Volker; Meder, Norbert; Uhlendorff, Uwe; Allemann-Ghionda, Cristina \& Mertens, Gerhard (Hrsg.): Handbuch der Erziehungswissenschaft. Verlag Ferdinand Schöningh, 581-619.

Winkler, Michael (2013): Kritische Soziale Arbeit - Anmerkungen zu Möglichkeiten und Grenzen einer Idee. In: Hünersdorf, Bettina \& Hartmann, Jutta (Hrsg.): Was ist und wozu betreiben wir Kritik in der Sozialen Arbeit? Wiesbaden: Springer Fachmedien Wiesbaden, 145-164.

Winkler, Michael (2021): Eine Theorie der Sozialpädagogik: Neuausgabe mit einem neuen Nachwort. Herausgegeben von Gaby Flösser und Marc Witzel. Weinheim: Juventa Verlag.

Witzel, Marc (2015): Digitale Medien in der stationären Erziehungshilfe zwischen lebensweltlichen und institutionellen Kontexten. In: Kutscher, Nadia; Ley, Thomas \& Seelmeyer, Udo (Hrsg.): Mediatisierung (in) der Sozialen Arbeit. Baltmannsweiler: Schneider Hohengehren, 115-129.

Zillien, Nicole (2009): Digitale Ungleichheit: Neue Technologien und alte Ungleichheiten in der Informations- und Wissensgesellschaft. 2. Auflage. Wiesbaden: VS Verlag für Sozialwissenschaften. 